\title{
MAGNETIC MOMENT AND ELECTRONIC STRUCTURE OF AMORPHOUS ALLOYS OF THE TYPE $\mathrm{Fe}_{80-x} \mathrm{TM}_{x} \mathrm{~B}_{20}$
}

\author{
E. NOWAKOWSKA \\ Warsaw Agriculture University, Rakowiecka 26/30, 02-528 Warszawa, Poland \\ W. Dudek, J. Oleniacz and W. ZYCH \\ Institute of Physics, Warsaw Technical University \\ Koszykowa 75, 00-662 Warszawa, Poland \\ (Received July 5, 1994)
}

\begin{abstract}
Magnetic properties of amorphous alloys of the type $\mathrm{Fe}_{80-x} \mathrm{TM}_{x} \mathrm{~B}_{20}$ $(0 \leq x \leq 20$ at. $\%, \mathrm{TM}=\mathrm{Ni}, \mathrm{Co}, \mathrm{Mn}, \mathrm{Cr}, \mathrm{V}, \mathrm{Ti})$ are presented and analyzed. The basis of our research work was the Mössbauer measurements, magnetic saturation measurements, derivative thermo-magnetogravimetry and differential thermal analysis measurements. The magnetic moments are discussed in terms of Friedel's virtual-bound-state model for light TM impurities in strong ferromagnets. Some conclusions about the electronic structure are presented.
\end{abstract}

PACS numbers: $76.80 .+\mathrm{y}, 75.50 . \mathrm{Bb}$

\section{Introduction}

The concentration dependence of the magnetic moment in amorphous alloys, where transition metal substitutes for another transition metal, was studied by many authors. It has been found that in amorphous $\mathrm{Fe}_{80} \mathrm{~B}_{20}$ alloys the magnetic moment per metallic atom follows the Slater-Pauling curve when $\mathrm{Ni}$ or Co substitutes for $\mathrm{Fe}$, while strong departures are observed for $\mathrm{Mn}, \mathrm{Cr}, \mathrm{V}$, or Ti substituted alloys. In this case the behavior of the magnetic moment can be understood in terms of Friedel's virtual-bound-state model [1].

A new way of plotting magnetization data was proposed in Ref. [2]. The new construction differs from the usual Slater-Pauling plot in the replacement of electron-to-atom ratio by a related but different quantity, which is called "magnetic valence". In this model several alloy systems which appear in the usual Slater-Pauling plot as departures from regular behavior become regular in the 
new plot. Because the generalized Slater-Pauling model embodies the Friedel theory and the extension of the Friedel theory by Terakura and Kanamori [21], a wide variety of the amorphous alloys can be studied by this method. Nevertheless, even this generalized Slater-Pauling model cannot explain the details of the moment variation in the amorphous alloys with metalloid content.

Analysis of the data within the framework of Friedel's model was made for the amorphous $\mathrm{Co}_{80-x} \mathrm{TM}_{x} \mathrm{~B}_{20}$ alloys with $\mathrm{TM}=\mathrm{Fe}, \mathrm{Mn}, \mathrm{Cr}, \mathrm{V}$ by O'Handley $[3,4]$ and for the amorphous $\left(\mathrm{Fe}_{100-x} \mathrm{TM}_{x}\right)_{79} \mathrm{P}_{13} \mathrm{~B}_{8}$ alloys with $\mathrm{TM}=\mathrm{Mn}, \mathrm{Cr}, \mathrm{V}$ by Durand $[5,6]$. We report here concentration dependence of the magnetic moment for the amorphous $\mathrm{Fe}$-based alloys with boron as the only metalloid.

\section{Experimental}

Metallic glasses of $35 \mu \mathrm{m}$ thickness were produced by the melt quenching technique. Each foil was checked by X-ray spectroscopy to make sure that no crystalline phase was present. Simultaneous derivative thermo-magnetogravimetry differential thermal analysis (DTMG-DTA) method was applied to find the Curie temperature and crystallization temperature. The Mössbauer measurements were performed with a spectrometer operating in a constant acceleration mode. The source was $\mathrm{Co}^{57}$ in $\mathrm{Cr}$ matrix. Standard method for magnetization measurements has been used. Some of these results have already been published [7-16].

The magnetic moments at room temperature have been estimated by combining the results of the Mössbauer measurements with bulk magnetization measurements. From the average hyperfine field on ${ }^{57} \mathrm{Fe}$ one can obtain the mean magnetic moment of $\mathrm{Fe}\left(\mu_{\mathrm{Fe}}\right)$ and from magnetization - the mean magnetic moment of metals $(\mathrm{Fe}+\mathrm{TM})$ in the alloy as a whole $(\bar{\mu})$. In order to obtain the mean magnetic moment of the transition metal $\mu_{\mathrm{TM}}$ we can use the relation

$$
\bar{\mu}=(1-c) \mu_{\mathrm{Fe}}+\mu_{\mathrm{TM}} c,
$$

where $c$ is the relative TM concentration $(c=x / 80$ in the notation of $\left.\left(\mathrm{Fe}_{1-c} \mathrm{TM}_{c}\right)_{80} \mathrm{~B}_{20}\right)$.

\section{Results}

The variation of the mean magnetic moment per metal atom $\bar{\mu}$ as a function of $x$ is shown in Fig. 1. Assuming that for small concentration $(x \leq 6$ at.\%) this variation is linear, we can describe $\bar{\mu}(c)$ by the empirical expression

$$
\bar{\mu}=\mu_{\mathrm{Fe}}+\xi c \mu_{\mathrm{B}},
$$

where $\xi$ is the slope of the moment vs. TM content curve, and $\mu_{\mathrm{B}}$ is the Bohr magneton. We can observe that for $x \leq 6$ at.\% TM addition causes drop in the average moment per transition metal atom (Table). In the case of $\mathrm{Co}$ and $\mathrm{Ni}$ it is a relatively small effect. $\mathrm{Cr}$ and $\mathrm{V}$ cause $\mu$ to decrease nearly twice as fast as $\mathrm{Ti}$ and $\mathrm{Mn}$. Such dependence has also been observed for crystalline (TM, Fe) ${ }_{2} \mathrm{~B}$ compounds $(\mathrm{TM}=\mathrm{Mn}, \mathrm{Cr}, \mathrm{V}, \mathrm{Ti})[19]$.

There are no values of $\bar{\mu}$ for $\mathrm{V}$ and $\mathrm{Cr}$ containing amorphous alloys, because these alloys are paramagnetic for $x>20$ at.\% at room temperature. 


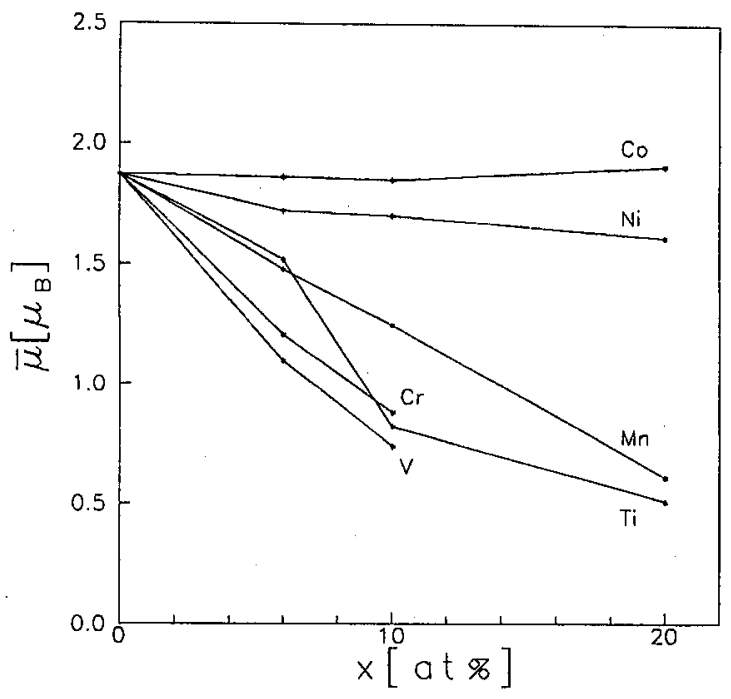

Fig. 1. Concentration dependence of the mean magnetic moment for amorphous $\mathrm{Fe}_{80-x} \mathrm{TM}_{x} \mathrm{~B}_{20}$ alloys.

\section{TABLE}

Effect of TM substitutions for Fe in $\mathrm{Fe}_{80} \mathrm{~B}_{20}$ amorphous alloys according to Eqs. (5), (6).

\begin{tabular}{c|c|c|c|c}
\hline \hline & $Z$ & \multicolumn{1}{c|}{$\xi$} & \multicolumn{1}{c}{$Z_{\uparrow}$} & $Z^{\downarrow}$ \\
\hline $\mathrm{Ni}$ & +2 & -2.00 & 0.00 & +2.00 \\
$\mathrm{Co}$ & +1 & -0.13 & +0.44 & +0.56 \\
$\mathrm{Mn}$ & -1 & -5.20 & -3.10 & +2.10 \\
$\mathrm{Cr}$ & -2 & -8.80 & -5.10 & +3.10 \\
$\mathrm{~V}$ & -3 & -10.30 & -6.70 & +3.70 \\
$\mathrm{Ti}$ & -4 & -4.70 & -4.40 & +0.40
\end{tabular}

The Curie temperature is plotted as a function of TM concentration in Fig. 2. Concentration dependence of $\bar{\mu}$ and $T_{\mathrm{C}}$ is opposite for $\mathrm{Ni}$ and Co. Addition of $\mathrm{Co}$ and $\mathrm{Ni}$ increases $T_{\mathrm{C}}$. The remaining solutes cause $T_{\mathrm{C}}$ to decrease.

The positive and equal to zero gradients in Co and Ni respectively substituted amorphous alloys are observed for the concentration dependence of the mean magnetic moment of $\mathrm{Fe}-\mu_{\mathrm{Fe}}(c)$ (Fig. 3). The data of Fig. 2 and Fig. 3 may be correlated by accepting a few assumptions about the relative populations of majority and minority spin states as was done by O'Handley [17]. 


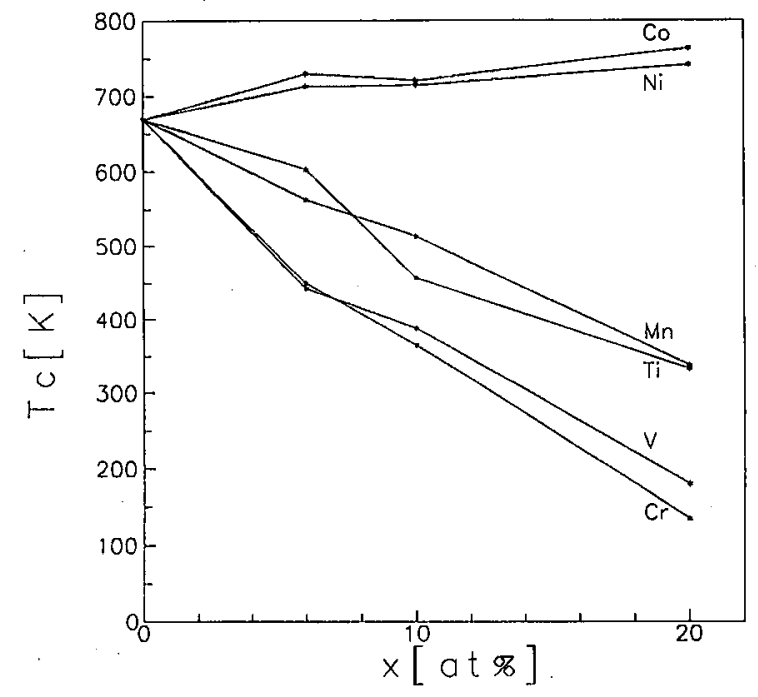

Fig. 2. Concentration dependence of the Curie temperature for amorphous $\mathrm{Fe}_{80-x} \mathrm{TM}_{x} \mathrm{~B}_{20}$ alloys.

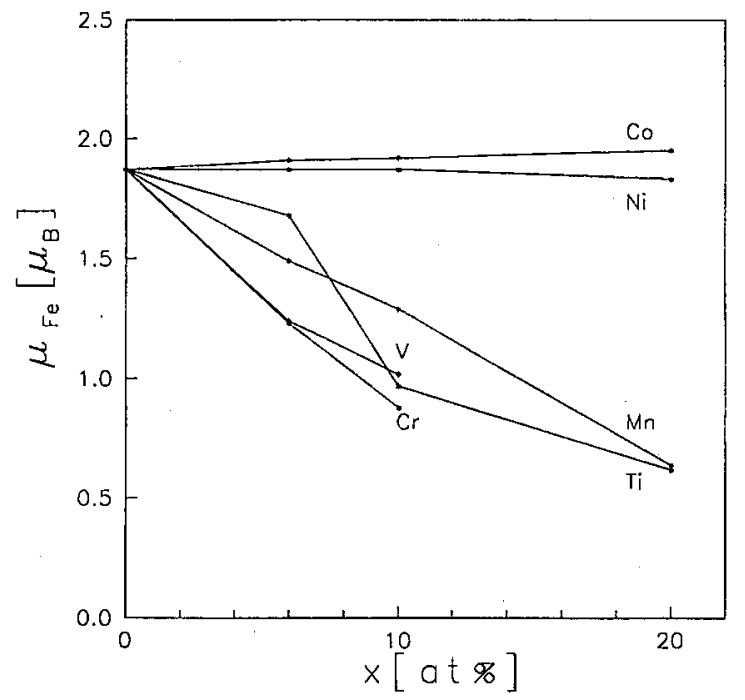

Fig. 3. Concentration dependence of the mean magnetic moment of Fe for amorphous $\mathrm{Fe}_{80-x} \mathrm{TM}_{x} \mathrm{~B}_{20}$ alloys.

\section{Interpretation}

It is well known that for $\mathrm{Fe}-\mathrm{Co}$ and $\mathrm{Fe}-\mathrm{Ni}$ amorphous alloys the variation of the mean magnetic moment per metal atom $\bar{\mu}$ as a function of the average outer electron concentration of metallic atoms $\bar{N}$ follows the Slater-Pauling curve:

$$
\bar{\mu}=\mu_{\mathrm{Fe}}-\bar{N} \text {. }
$$


Alloys of Fe with solute elements on the left of the transition series like $\mathrm{Mn}, \mathrm{Cr}, \mathrm{V}$, Ti, exhibit strong departure from the law expressed by Eq. (3). Our experimental results plotted as a function of $\bar{N}$ are shown in Fig. 4.

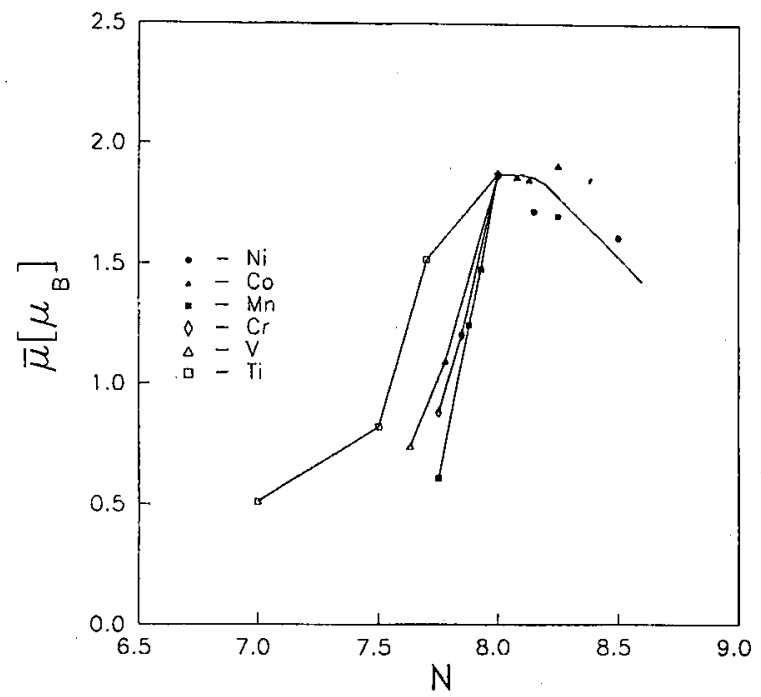

Fig. 4. The mean magnetic moment $\bar{\mu}$, plotted as a function of the average outer electron concentration $\bar{N}$ of transition metals. by

Equation (3) is in good agreement with the rigid-band approximation given

$$
\bar{\mu}=\mu_{\mathrm{Fe}}-Z c \mu_{\mathrm{B}},
$$

where $Z$ is the atomic number difference of the solute relative to the matrix. The rigid-band approximation applies only for small concentrations of impurities that weakly perturb the periodic potential of the matrix $(c \ll 1,|Z|=1)$. This approximation fails for $|Z| \geq 2$ as in the case of $\mathrm{Mn}, \mathrm{Cr}, \mathrm{V}$, Ti. Friedel's virtual-bound-state (v-b-s) model [1] can be used in order to account for sharply decreasing moments of these alloys, containing light transition metal solutes. In this model the variation of the mean magnetic moment $\bar{\mu}(c)-$ for $c \ll 1-$ is given by

$$
\bar{\mu}(c)=\mu_{\mathrm{Fe}}+\left(Z_{\uparrow}-Z^{\downarrow}\right) c \mu_{\mathrm{B}},
$$

where $Z_{\uparrow}$ and $Z^{\downarrow}$ are displaced charge by one impurity in the band of spin $\uparrow$ or $\downarrow$ respectively. From the Friedel sum rule, $Z_{\uparrow}$ and $Z \downarrow$ are related to the impurity excess charge $Z$ by

$$
Z=Z_{\uparrow}+Z^{\downarrow}
$$

The experimental values of the initial slopes $\xi=\mathrm{d} \bar{\mu} / \mathrm{d} c$ with Eqs. (5), (6) allow the determination of the screening charge in each group of bands with given spin and can be interpreted in terms of the electronic structure. Table contains the 
data of Fig. 1 obtained on the basis of Eqs. (5), (6). Experimental values for $\xi$ are calculated for low concentrations $(x \leq 6$ at.\%).

In the case of Co and $\mathrm{Ni}, Z_{\uparrow}$ is about zero and the variation of the mean magnetic moment is given by Eq. (4). In a simple model with nonhybridized $s-d$ bands, the total displaced charge is zero if the $d_{\uparrow}$ band is completely filled. The $Z$ supplementary electrons of each impurity are going mostly to the $d^{\downarrow}$ band because the density of states at the Fermi level is much higher for the $d$ band than for the $s$ band. Because the screening in the $d_{\uparrow}$ bands is very small for Co and $\mathrm{Ni}$, the amorphous $\mathrm{Fe}_{80} \mathrm{~B}_{20}$ matrix can be regarded as a strong ferromagnet in the sense described above. For $\mathrm{Mn}, \mathrm{Cr}, \mathrm{V}, \mathrm{Ti}, Z_{\uparrow}$ is negative and decreases to about -5 with increasing values of $|Z|$. These elements have nuclear charges much smaller than the matrix (large negative $Z$ ), and introduce a perturbation which is strongly repulsive. When the impurity potential is sufficiently repulsive, $d$ bound states are repelled from the filled $d_{\uparrow}$ band and these states are emptied when their energy is larger than the Fermi energy. Neglecting the host $s-d$ hybridization, a non-zero value of $Z_{\uparrow}$ can only occur if a perturbed $d_{\uparrow}$ state is unoccupied. The number $n$ of electrons within the corresponding virtual bound states can be estimated from $Z_{\uparrow}$ by the equation

$$
n=Z_{\uparrow}+5 \text {. }
$$

$\mathrm{Cr}, \mathrm{V}, \mathrm{Ti}$ additions behave more in accordance with the expectation of the $\mathrm{v}$-b-s model for strongly repulsive solutes. The $3 d_{\uparrow} \mathrm{v}$-b-s is above the Fermi energy and its charge is displaced to the $3 d^{\downarrow}$ band suppressing the moments around the impurity site. $\mathrm{Mn}$ is intermediate, implying that its low lying $3 d_{\uparrow} \mathrm{v}-\mathrm{b}-\mathrm{s}$ intersects the Fermi level because the displaced charge $Z_{\uparrow}$ is significantly smaller than 5 .

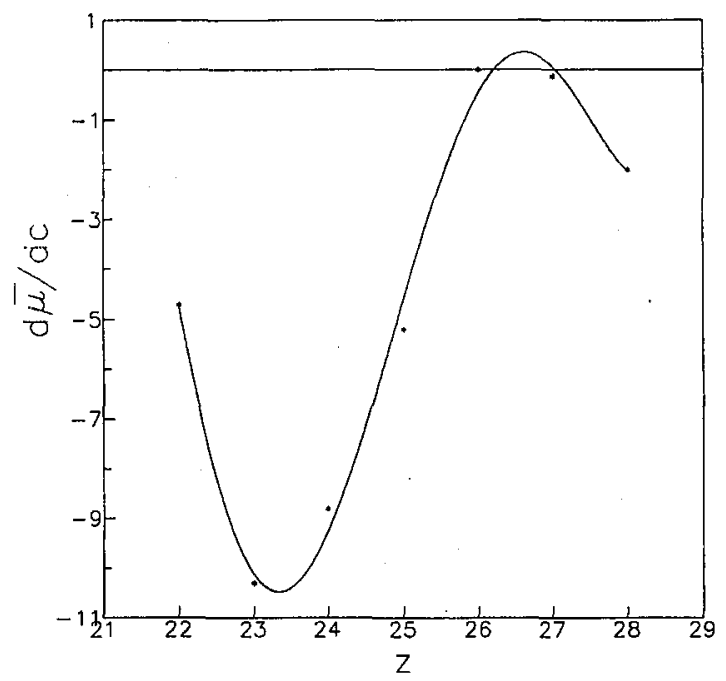

Fig. 5. The dependence of the mean magnetic moment increase $d \bar{\mu} / \mathrm{d} c$ on the atomic number $Z$ of TM-substituting Fe atoms in amorphous $\mathrm{Fe}_{80-x} \mathrm{TM}_{x} \mathrm{~B}_{20}$ alloys. 


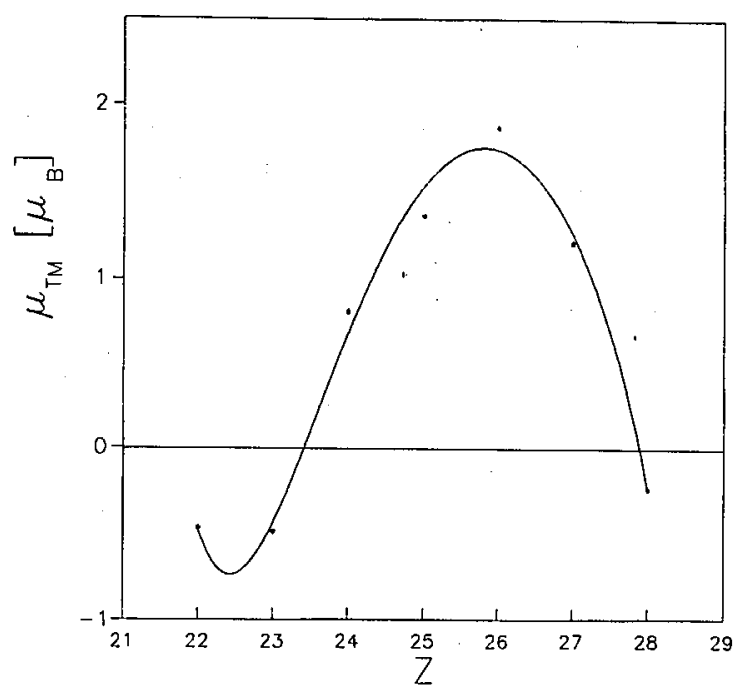

Fig. 6. The dependence of the impurity magnetic moment on the atomic number $Z$ of TM-substituting Fe atoms in amorphous $\mathrm{Fe}_{80-x} \mathrm{TM}_{x} \mathrm{~B}_{20}$.

Thus, the v-b-s are half filled for $\mathrm{Mn}$ and practically empty for $\mathrm{Cr}, \mathrm{V}$ and $\mathrm{Ti}$ in amorphous $\mathrm{Fe}_{80} \mathrm{~B}_{20}$.

Figure 5 presents the dependence of the mean magnetic moment increase $\mathrm{d} \bar{\mu} / \mathrm{d} c$ and Fig. 6 - the dependence of the impurity magnetic moment $\mu_{\mathrm{TM}}$ on the atomic number $Z$ of TM-substituting Fe atoms in iron alloys. Figure 6 shows a change of sign of the local magnetic moment on the impurity state. This feature can be explained in strong ferromagnets by the existence of the $v-b-s$ and intersection of the v-b-s with the Fermi level [18].

Our conclusions are similar to those obtained by Durand $[5,6]$.

The comparison with the crystalline compounds done in [6] suggests that the band structure of the amorphous $\mathrm{Fe}_{80} \mathrm{~B}_{20}$ are much more similar to crystalline $\mathrm{Fe}_{3} \mathrm{P}, \mathrm{Fe}_{3} \mathrm{C}$ and $\mathrm{Fe}_{2} \mathrm{~B}$ compounds than that of $\alpha \mathrm{Fe}$, which is known to be a weak ferromagnet.

The same conclusions were obtained on the basis of the analysis of the concentration dependence of the Curie temperature [19]. In the framework of the band model of ferromagnetism, the Curie temperature is determined by the electronic structure. The similarities and differences between the Curie temperature of various alloys can be taken as an indication of respective similarities and differences between their electronic structure. The main feature of the data presented in Ref. [20] is the similarity between the Ni- and Co-based crystalline and Fe-based amorphous alloys and quite different character of the Fe-based crystalline alloys data. Our experimental results on the relative $T_{\mathrm{C}}$ change in $\mathrm{Fe}_{80} \mathrm{~B}_{20}$ due to transition metal additions (Fig. 7) are similar to those reported by Donald [20] and suggest a full majority sub-band in Fe-based amorphous alloys.

In Fig. 8 generalized Slater-Pauling curve (the mean magnetic moment per metal atom versus average magnetic valence) was plotted on the basis of our 


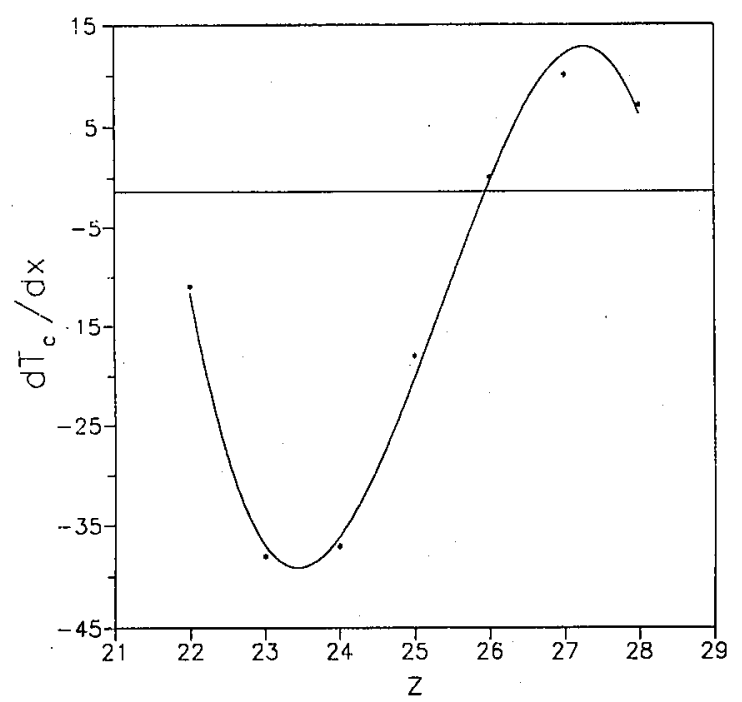

Fig. 7. The dependence of the Curie temperature increase $\mathrm{d} T_{\mathrm{C}} / \mathrm{d} x$ on the atomic number $Z$ of TM-substituting Fe atoms in amorphous $\mathrm{Fe}_{80-x} \mathrm{TM}_{x} \mathrm{~B}_{20}$.

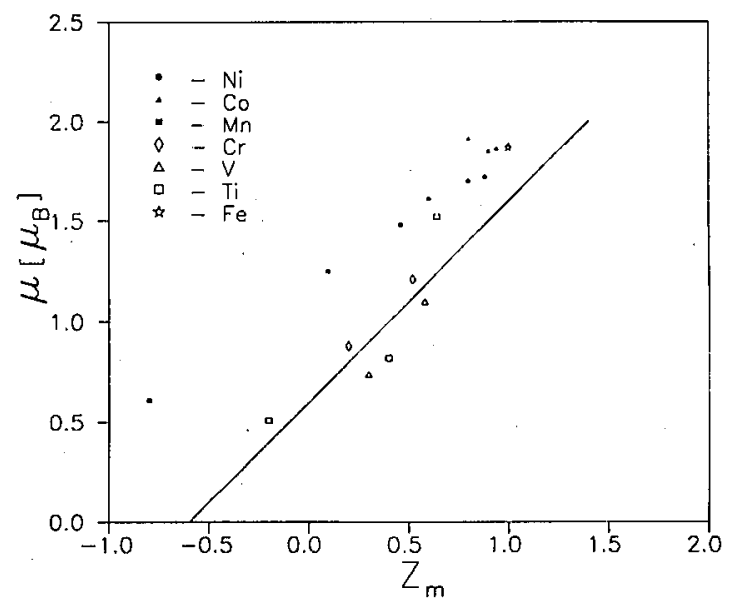

Fig. 8. The dependence of the mean magnetic moment plotted as a function of the average magnetic valence.

experimental data. Magnetic valence $Z_{\mathrm{m}}$ defined as

$$
Z_{\mathrm{m}}=2 N_{d \dagger}-Z
$$

where $N_{d \dagger}$ is the number of electrons in the majority spin band and $Z$ is equal to the negative value of the valence charge $Z$, except for the $\mathrm{Fe}, \mathrm{Co}$ and $\mathrm{Ni}$ column, for which $2 N_{d 1}=10$ gives $Z_{\mathrm{m}}=2,1$, and 0 respectively.

The $45^{\circ}$ line corresponds to a fixed number (0.3) of $s p$ up-spin electrons and 
the magnetization $M$ (in units of $\mu_{\mathrm{B}}$ ) is given by

$$
M=Z_{\mathrm{m}}+0.6 \text {. }
$$

As a general rule, the authors of Ref. [2] expect alloys, whose magnetization falls below the $M=Z_{\mathrm{m}}+0.6$ line, to be magnetically weak. Figure 8 suggests that the amorphous alloys of the type $\mathrm{Fe}_{1-x} \mathrm{TM}_{x} \mathrm{~B}_{20}$ are magnetically strong. The data points tend to follow a slightly displaced straight line. This is in agreement with the results of the band calculations [21] - whereas the magnetization of the elements tends to follow the $M=Z_{\mathrm{m}}+0.6$ line, the magnetization of alloys should follow a slightly displaced straight line $M=Z_{\mathrm{m}}+0.9$.

In summary, as we have seen, the magnetic moment of $\mathrm{Fe}_{80-x} \mathrm{TM}_{x} \mathrm{~B}_{20}$ amorphous alloys can be interpreted in terms of Friedel's v-b-s model. On the basis of this analysis some information about the electronic structure of $\mathrm{Fe}_{80} \mathrm{~B}_{20}$ and of the impurities of transition metals can be obtained. Our experimental results suggests a full majority sub-band in $\mathrm{Fe}_{80} \mathrm{~B}_{20}$ amorphous alloys. Transition metal solutes lighter than iron cause a $\mathrm{v}-\mathrm{b}$-s to appear above the $d$ band of the $\mathrm{Fe}-\mathrm{B}$ matrix. In the case of $\mathrm{Cr}, \mathrm{V}, \mathrm{Ti}$ the $3 d_{\uparrow} \mathrm{v}$-b-s is above the Fermi level and is empty, while for Mn the $3 d_{\uparrow}$ v-b-s intersects the Fermi level.

\section{References}

[1] J. Friedel, Nuovo Cim. Suppl. to Vol. 3 (1958).

[2] A.R. Williams, V.L. Moruzzi, A.P. Malozemoff, K. Terakura, IEEE Trans. Magn. Mag-19, 1983 (1983).

[3] R.C. O'Handley, Solid State Commun. 38, 703 (1981).

[4] R.C. O'Handley, M.O. Sullivan, J. Appl. Phys. 52, 1841 (1981).

[5] J. Durand, Amorphous Magnetism II, Eds. Levy, Hasegava, Plenum Press, New York 1977, p. 305.

[6] J. Durand, C. Thompson, A. Amamou, Rapidly Quenched Metals III, Ed. B. Cantor, The Metals Soc., London 1978, p. 109.

[7] M. Eukasiak, H. Matyja, W. Nazarewicz, J. Pluta, I. Śledzińska, J. Suwalski, W. Zych, Acta Phys. Pol. A 68, 199 (1985).

[8] W. Zych, J. Milczarek, Phys. Status Solidi A 90, K165 (1985).

[9] R. Moskalewicz, W. Zych, Phys. Status Solidi A 97, K43 (1986).

[10] W. Zych, Phys. Status Solidi A 99, K125 (1987).

[11] R. Moskalewicz, W. Zych, Acta Phys. Pol. A 72, 185 (1987).

[12] J. Gwiazda, M. Peryt, W. Peryt, W. Zych, E. Mariańska, Acta Phys. Pol. A 72; 165 (1987).

[13] J. Gwiazda, E. Mariańska, W. Zych, Phys. Status Solidi A 108, K125 (1988).

[14] J. Gwiazda, E. Mariańska, J. Oleniacz, M. Peryt, W. Zych, Hyperfine Interact. 55, 973 (1990).

[15] W. Dudek, J. Gwiazda, E. Mariańska, J. Oleniacz, W. Zych, J. Magn. Magn. Mater. 86, 243 (1991).

[16] W. Dudek, J. Gwiazda, E. Mariańska, J. Oleniacz, W. Zych, J. Magn. Magn. Mater. 94, 243 (1991). 
[17] R.C. O'Handley, D.S. Boudreaux, Phys. Status Solidi A 44, 607 (1978).

[18] F. Gautier, Ann. Phys. 8, 251 (1973-1974).

[19] M.C. Cadeville, E. Daniel, J. Phys. (Paris) 27, 449 (1966).

[20] I.W. Donald, T. Kemeny, H.A. Davies, J. Phys. F, Metal Phys. 11, L-131 (1981).

[21] K. Terakura, J. Kanamori, Prog. Theor. Phys. 46, 1007 (1971). 